ARTICLE

\title{
Dynamic active-site generation of atomic iridium stabilized on nanoporous metal phosphides for water oxidation
}

Kang Jiang ${ }^{1}$, Min Luo ${ }^{2}$, Ming Peng ${ }^{1}$, Yaqian Yu', Ying-Rui Lu ${ }^{3}$, Ting-Shan Chan ${ }^{3}$, Pan Liu ${ }^{4}$, Frank M. F. de Groot ${ }^{5}$ \& Yongwen Tan (10) ${ }^{1 \times}$

Designing efficient single-atom catalysts (SACs) for oxygen evolution reaction (OER) is critical for water-splitting. However, the self-reconstruction of isolated active sites during OER not only influences the catalytic activity, but also limits the understanding of structureproperty relationships. Here, we utilize a self-reconstruction strategy to prepare a SAC with isolated iridium anchored on oxyhydroxides, which exhibits high catalytic OER performance with low overpotential and small Tafel slope, superior to the $\mathrm{IrO}_{2}$. Operando X-ray absorption spectroscopy studies in combination with theory calculations indicate that the isolated iridium sites undergo a deprotonation process to form the multiple active sites during OER, promoting the $\mathrm{O}-\mathrm{O}$ coupling. The isolated iridium sites are revealed to remain dispersed due to the support effect during OER. This work not only affords the rational design strategy of OER SACs at the atomic scale, but also provides the fundamental insights of the operando OER mechanism for highly active OER SACs.

\footnotetext{
${ }^{1}$ College of Materials Science and Engineering, Hunan University, Changsha, Hunan 410082, China. ${ }^{2}$ Department of Physics, Shanghai Polytechnic University, Shanghai 201209, China. ${ }^{3}$ National Synchrotron Radiation Research Center, Hsinchu 300, Taiwan. ${ }^{4}$ State Key Laboratory of Metal Matrix Composites, School of Materials Science and Engineering, Shanghai Jiao Tong University, Shanghai 200030, China. ${ }^{5}$ Inorganic Chemistry \& Catalysis, Debye Institute for Nanomaterials Science, Utrecht University, Universiteitsweg 99, 3584 CGUtrecht, The Netherlands. ${ }_{\text {email: tanyw@hnu.edu.cn }}$
} 
E lectrocatalytic water splitting for hydrogen and oxygen production provides an attractive path to obtain sustainable energy via the conversion and storage of intermittent solar and wind energies ${ }^{1,2}$. However, the bottleneck in improving water electrolysis is mainly caused by sluggish reaction kinetics at the anode, where water is oxidized and the oxygen evolution reaction (OER) occurs ${ }^{2,3}$. Currently, precious metal oxides, iridium (Ir), and ruthenium $(\mathrm{Ru})$ oxides are the highly efficient OER electrocatalysts but suffer from relative scarcity and high-cost ${ }^{4}$. Though great efforts have been made to develop the earth-abundant transition-metalbased catalysts, including metal oxides or hydroxides ${ }^{5-14}$, borides $^{15,16}$, phosphides ${ }^{17-21}$, sulfides ${ }^{22,23}$, and selenides ${ }^{24,25}$, as low-cost alternative for OER. However, their catalytic performance remains far from satisfactory. Thus, it is highly desirable to improve these OER systems, thus achieving high energy efficiency and costeffectiveness in alkaline electrolysis.

Single-atom catalysts (SACs) offer a favorable pathway to maximize the catalytic activity while significantly reducing the amount of metals for chemical reactions ${ }^{26-32}$. The introduction of single atoms can largely improve the OER performance of support materials, such as carbon materials ${ }^{27-30}$, metals ${ }^{31}$, and metal oxide $^{32}$. However, using metal phosphides as a support to anchor single-atom are still rare. They undergo surface self-reconstruction to form oxyhydroxides under OER conditions, providing abundant oxygen ligands for the anchoring of isolated atoms ${ }^{18,19}$. A fact that cannot be neglected is that most active sites on the surface of catalyst would undergo structural self-reconstruction due to the electro-derived structural oxidation processes ${ }^{4,33}$. Accordingly, it is reasonable to believe that the structural self-reconstruction of active sites would change the catalytic activity, especially for SACs. For instance, the aggregation of isolated atoms under extreme OER condition will lead to dramatic changes in the properties of catalysts. Therefore, a major challenge in the development of SACs is the difficulty to stabilize isolated atoms under drastic reaction conditions. Besides, stable SACs possessing the well-defined active centres offer an ideal model for exploring the origin of the activity under drastic OER conditions ${ }^{34,35}$. However, directly monitoring the dynamic behaviors of isolated active sites and identifying the synergy between different sites under extreme OER conditions are rarely available for SACs ${ }^{28,30}$, yet are pivotal for the development of efficient OER catalysts ${ }^{35}$. Consequently, it is highly desirable to develop a stable SACs with efficient OER performance and mechanistic insight into the single-atoms induced enhancement of OER activity.

In this work, we adopt a facile self-reconstruction strategy for single-atom Ir catalysts with controllable deposition of isolated Ir atoms on free-standing nanoporous $\left(\mathrm{Ni}_{0.74} \mathrm{Fe}_{0.26}\right)_{3} \mathrm{P}$ (denoted as np$\mathrm{Ir} / \mathrm{NiFeP}$ ). After subsequent electrochemical activation, the isolated Ir atoms turn into a stable state with higher valence and more oxygen ligands. The optimal catalyst (denoted as np-Ir/NiFeO) exhibits a high OER performance with low overpotential, high mass activity, and long stability, much superior to commercial $\mathrm{IrO}_{2}$ and most previously reported catalysts. Significantly, we identify, by employing operando X-ray absorption spectroscopy (XAS) and density functional theory (DFT) calculations, that the isolated Ir atoms undergo a variation from the single active site to multiple active sites through a deprotonation process during the OER process, which are responsible for the superior catalytic performance. Furthermore, the surface $\mathrm{Ni}(\mathrm{Fe})$ oxyhydroxides can form a shrinkage structure during OER conditions, further fixing the isolated Ir atoms, causing the excellent stability.

\section{Results}

Material synthesis and characterization. The nanoporous $\left(\mathrm{Ni}_{0.74} \mathrm{Fe}_{0.26}\right)_{3} \mathrm{P}$ (denoted as $\mathrm{np}-\mathrm{NiFeP}$ ) was synthesized using an electrochemically selective etching method (see "Method" section and Supplementary Figs. 1, 2) ${ }^{36}$. Then, Ir was atomically deposited on np-NiFeP by cyclic voltammetry $(\mathrm{CV})$ using $\mathrm{IrCl}_{3}-\mathrm{KOH}$ as an electrolyte solution to produce the pre-catalyst (np-Ir/ NiFeP) (Supplementary Figs. 3, 4). After electrochemical activation in basic solution, np-Ir/NiFeP underwent surface selfreconstruction to further stabilize isolated Ir atoms, forming the desired catalyst (np-Ir/NiFeO) (Fig. 1a, see "Method" section, and Supplementary Fig. 5). X-ray diffraction (XRD) patterns (Supplementary Fig. 6) show that both $\mathrm{np}-\mathrm{Ir} / \mathrm{NiFeP}$ and $\mathrm{np}-\mathrm{Ir} / \mathrm{NiFeO}$ have the dominant peaks identical to those of pristine np-NiFeP without characteristic peaks of Ir, which confirms the absence of Ir nanoparticles. While no peaks for $\mathrm{Ni}(\mathrm{Fe}$ ) (oxy)hydroxides were detected in the XRD patterns of $\mathrm{np}-\mathrm{Ir} / \mathrm{NiFeO}$, revealing the formation of amorphous oxide layer. The $\mathrm{Ni} / \mathrm{Fe}$ ratio approaches $1 / 3$ in $\mathrm{np}-\mathrm{Ir} / \mathrm{NiFeP}$ (Supplementary Fig. 7), which correspond to the formation of stable Fe doped $\gamma-\mathrm{NiOOH}$ after electrochemical activation $^{19}$. Representative high-angle annular dark-field scanning transmission electron microscopy (HAADF-STEM) images (Fig. 1b and Supplementary Fig. 8) at the reconstructed surface confirm that isolated Ir atoms (bright dots) uniform dispersed on the surface of amorphous $\mathrm{Ni}(\mathrm{Fe})$ (oxy)hydroxides. The singleatomic nature of Ir atoms and the amorphous nature of the reconstructed surface are further confirmed by line-scanning intensity profile (Fig. 1c) and high-resolution TEM (HRTEM, Fig. 1d and Supplementary Fig. 9). The STEM energy dispersive $\mathrm{X}$-ray spectroscopy (EDX) elemental mapping shows the material is composed of $\mathrm{Ir}, \mathrm{Ni}, \mathrm{Fe}, \mathrm{P}$, and $\mathrm{O}$ elements (Supplementary Fig. 10), in which Ir is homogeneously distributed across the reconstructed surface (Fig. 1e).

The chemical composition and oxidation states of catalysts were analyzed by X-ray photoelectron spectroscopy (XPS). Figure if shows deconvolution of the Ir $4 \mathrm{f}$ core level spectra, which exhibits a doublet ( $\operatorname{Ir} 4 f_{7 / 2}$ and $4 f_{5 / 2}$ ) at $62.0 \mathrm{eV}$ and $65.0 \mathrm{eV}$ for $n p-\mathrm{Ir} / \mathrm{NiFeP}$, indicating Ir atoms carry positive-charge in relation to metallic Ir due to the electronic interaction between Ir atoms and support ${ }^{37}$. After surface self-reconstruction, the doublet of $n p-\mathrm{Ir} / \mathrm{NiFeO}$ exhibits a positive-shift compared with that of $\mathrm{Ir} / \mathrm{NiFeP}$, underscoring the higher Ir valence state of $n p-I r / N i F e O$. The core-level Ni $2 p$ XPS spectrum of np-NiFeP displays three peaks with binding energies of $852.8,856.0$, and $861.6 \mathrm{eV}$ assigned to $\mathrm{Ni}_{3} \mathrm{P}, \mathrm{Ni}$ oxide or hydroxide, and relevant satellite peak, respectively (Supplementary Fig. 11a $)^{20,38}$. Meanwhile, two peaks with binding energies located at 706.5 and $712.8 \mathrm{eV}$ in core-level Fe $2 p$ XPS spectrum of $\mathrm{np}-\mathrm{NiFeP}$ are ascribed to $\mathrm{Fe}_{3} \mathrm{P}$ and $\mathrm{Fe}$ oxide (Supplementary Fig. $11 \mathrm{~b})^{18}$. Compared with np-NiFeP, the $\mathrm{Ni} 2 p$ and $\mathrm{Fe} 2 p$ XPS spectra of np-Ir/NiFeP show a redistribution of more $\mathrm{Ni}(\mathrm{Fe})$ oxides or hydroxides from $\mathrm{Ni}_{3} \mathrm{P}\left(\mathrm{Fe}_{3} \mathrm{P}\right)$ and phosphate due to the presence of alkaline solution and the reduction trend of the cathodic voltage (Supplementary Fig. 11c) ${ }^{24}$. During the atomic deposition process, Ir species are easily trapped by oxygen ligands and stabilized thereupon, producing $n p-I r / \mathrm{NiFeP}^{39}$. After electrochemical activation, the Ni $2 p$ and Fe $2 p$ XPS spectra confirm that a mixture of $\mathrm{Ni}(\mathrm{Fe})$ (oxy)hydroxides evolves at the surface of the np$\mathrm{Ir} / \mathrm{NiFeO}$, which changes the coordination environment of isolated Ir atoms ${ }^{18}$.

The X-ray absorption near-edge structure (XANES) spectroscopy and extended X-ray absorption fine structure (EXAFS) spectroscopy were measured to further probe the electronic and coordination structures of the catalysts ${ }^{40}$. The white line intensity of $\mathrm{np}-\mathrm{Ir} / \mathrm{NiFeP}$ in $\mathrm{Ir} \mathrm{L}_{3}$-edge XANES spectrum is situated between those of $\mathrm{Ir}$ foil and $\mathrm{IrO}_{2}$, indicating a cationic environment (Fig. 1g) ${ }^{37}$. After electrochemical activation, the white line intensity of $\mathrm{np}-\mathrm{Ir} / \mathrm{NiFeO}$ increased, indicating the further oxidation of the Ir species. As shown in the Fourier transform of EXAFS (FT-EXAFS) spectra (Fig. 1h), np-Ir/NiFeP 
a

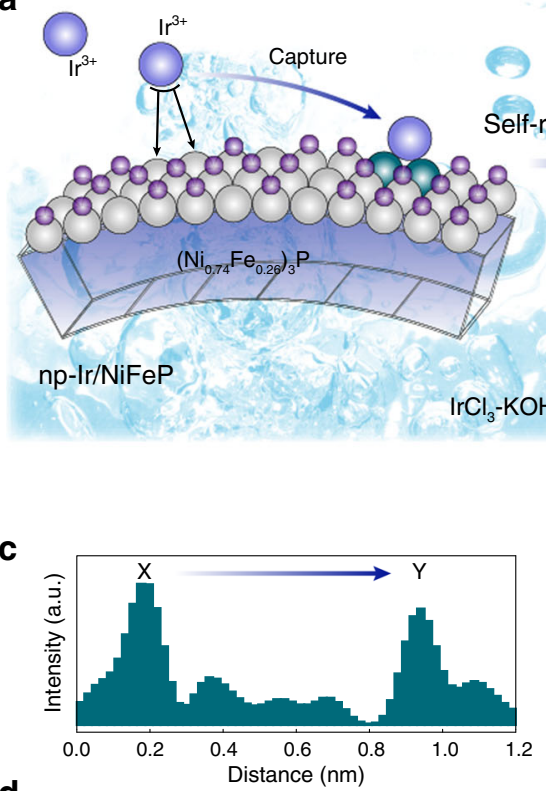

d

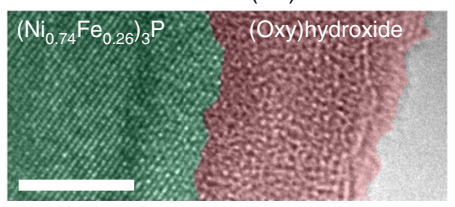

g

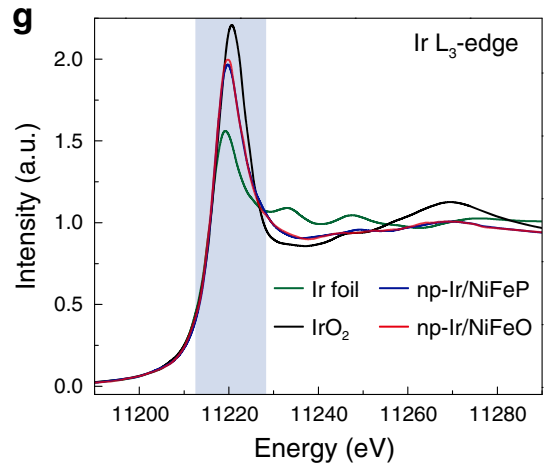

e
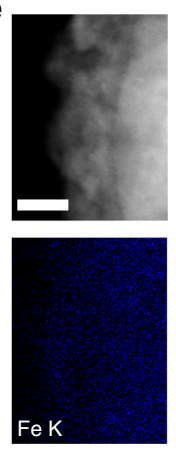

Ir M
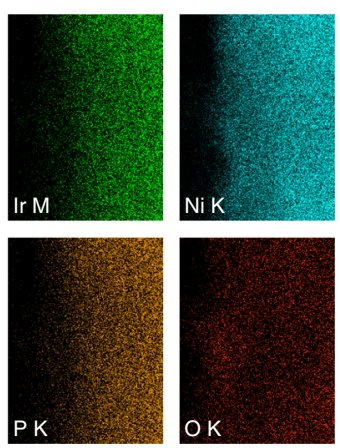

h

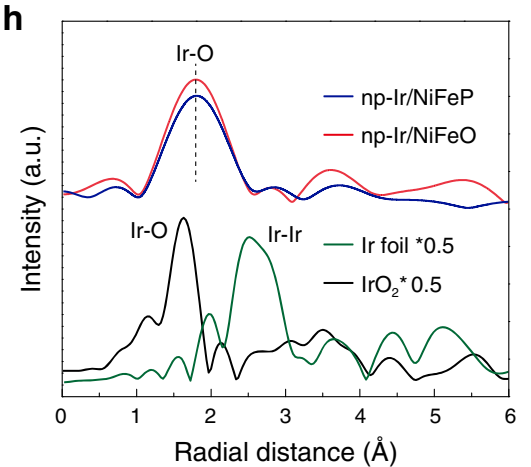

b

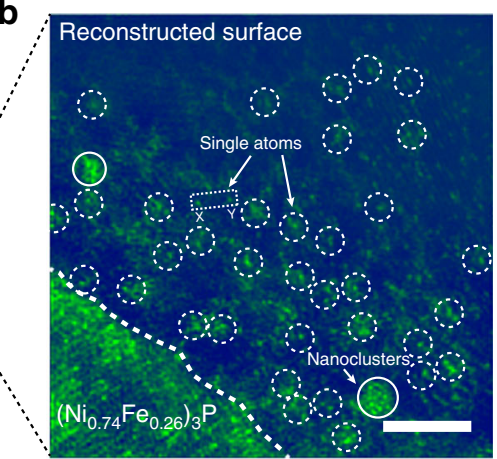

f

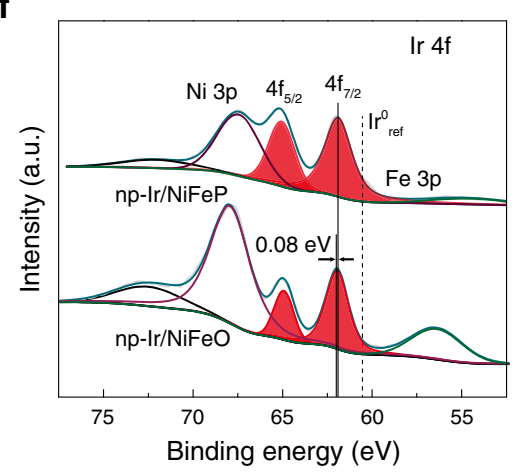

i

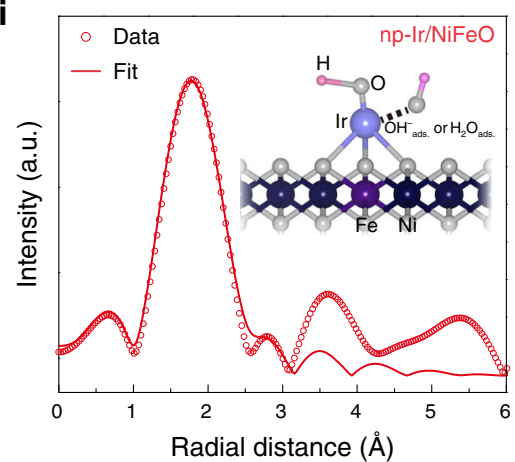

Fig. 1 Structural characterization of np-Ir/NiFeO. a Schematic illustration of the preparation of np-Ir/NiFeO. b HAADF-STEM image of np-Ir/NiFeO, showing that Ir atoms appear as bright spots (highlighted by the white circles) and dispersed on the amorphous surface. $\mathbf{c}$ Intensity profiles along the lines $\mathrm{X}-\mathrm{Y}$ in (b). $\mathbf{d}$ HRTEM image, showing the interface between $\left(\mathrm{Ni}_{0.74} \mathrm{Fe}_{0.26}\right)_{3} \mathrm{P}$ and amorphous (oxy)hydroxides. e HAADF-STEM image and the corresponding EDX elemental mapping. $\mathbf{f}$ XPS spectra of np-Ir/NiFeP and np-Ir/NiFeO in Ir $4 f$ region. $\mathbf{g}$ The normalized XANES at the Ir $\mathrm{L}_{3}$-edge of Ir foil, $\mathrm{IrO}_{2}$, np-Ir/NiFeP, and np-Ir/NiFeO. h Corresponding to FT-EXAFS spectra from (g). i First-shell (Ir-O) fitting of FT-EXAFS spectrum for np-Ir/NiFeO. Inset shows the structure of optimized np-Ir/NiFeO. Scale bar: b $2 \mathrm{~nm}$, d $5 \mathrm{~nm}$, e $5 \mathrm{~nm}$.

exhibits a prominent peak at $1.78 \AA$ corresponding to $\mathrm{Ir}-\mathrm{O}$ scattering feature ${ }^{37,41}$. No typical peaks for Ir-Ir contribution are observed, revealing the isolated dispersion of Ir atoms. Compared with np-Ir/NiFeP, the intensity of Ir-O shell increases in the FTEXAFS spectrum of $n p-\mathrm{Ir} / \mathrm{NiFeO}$, indicating that isolated $\mathrm{Ir}$ atoms turn into a more stable state with higher valence and more oxygen ligands after surface self-reconstruction (Supplementary Fig. 11d ${ }^{39}$. Moreover, FT-EXAFS fitting of $n p-\mathrm{Ir} / \mathrm{NiFeO}$ based the structure model in DFT (Fig. 1i and Supplementary Table 3) demonstrates that each $\mathrm{Ir}$ atom is coordinated by four $\mathrm{O}$ atoms from oxygen ligands and two $\mathrm{O}$ atoms from absorbed hydroxyl or water group ${ }^{30}$, further demonstrating the single-atomic nature of Ir. The Ni K-edge XANES spectra for np-NiFeP, np-Ir/NiFeP, and $n p-\mathrm{Ir} / \mathrm{NiFeO}$ are shown in Supplementary Fig. 12a. The white line intensity of $n p-I r / N i F e P$ is slightly higher than that of np-
$\mathrm{NiFeP}$, implying the partial oxidation of the $\mathrm{Ni}$ species and the formation of an oxygen-enriched surface which provides the anchoring ligand for the $\mathrm{Ir}$ species ${ }^{8,42}$. After the activation process, the absorption-edge of $n p-\mathrm{Ir} / \mathrm{NiFeO}$ shifts to higher energy, in combination with an increase in the white line intensity due to the formation of a $\mathrm{Ni}$ (oxy)hydroxide layer ${ }^{9}$. The corresponding FT-EXAFS spectrum of np-Ir/NiFeP shows a decrease of the peak at $\sim 1.95 \AA$ in comparison with that of $n p$ NiFeP (Supplementary Fig. 12b), which can be attributed to the break of $\mathrm{Ni}-\mathrm{P}$ bonds during the generating of $\mathrm{Ni}$ oxides or hydroxides. Furthermore, compared with np-Ir/NiFeP, the prominent peak of $\mathrm{np}-\mathrm{Ir} / \mathrm{NiFeO}$ shows a low- $R$ shift by $0.06 \AA$, along with the rise and broadening of the peak, which is ascribed to a contribution of $\mathrm{Ni}-\mathrm{O}$ bond from $\mathrm{Ni}$ (oxy)hydroxides that partial overlapped with the $\mathrm{Ni}-\mathrm{P}$ bond ${ }^{21}$. In addition, the peak 

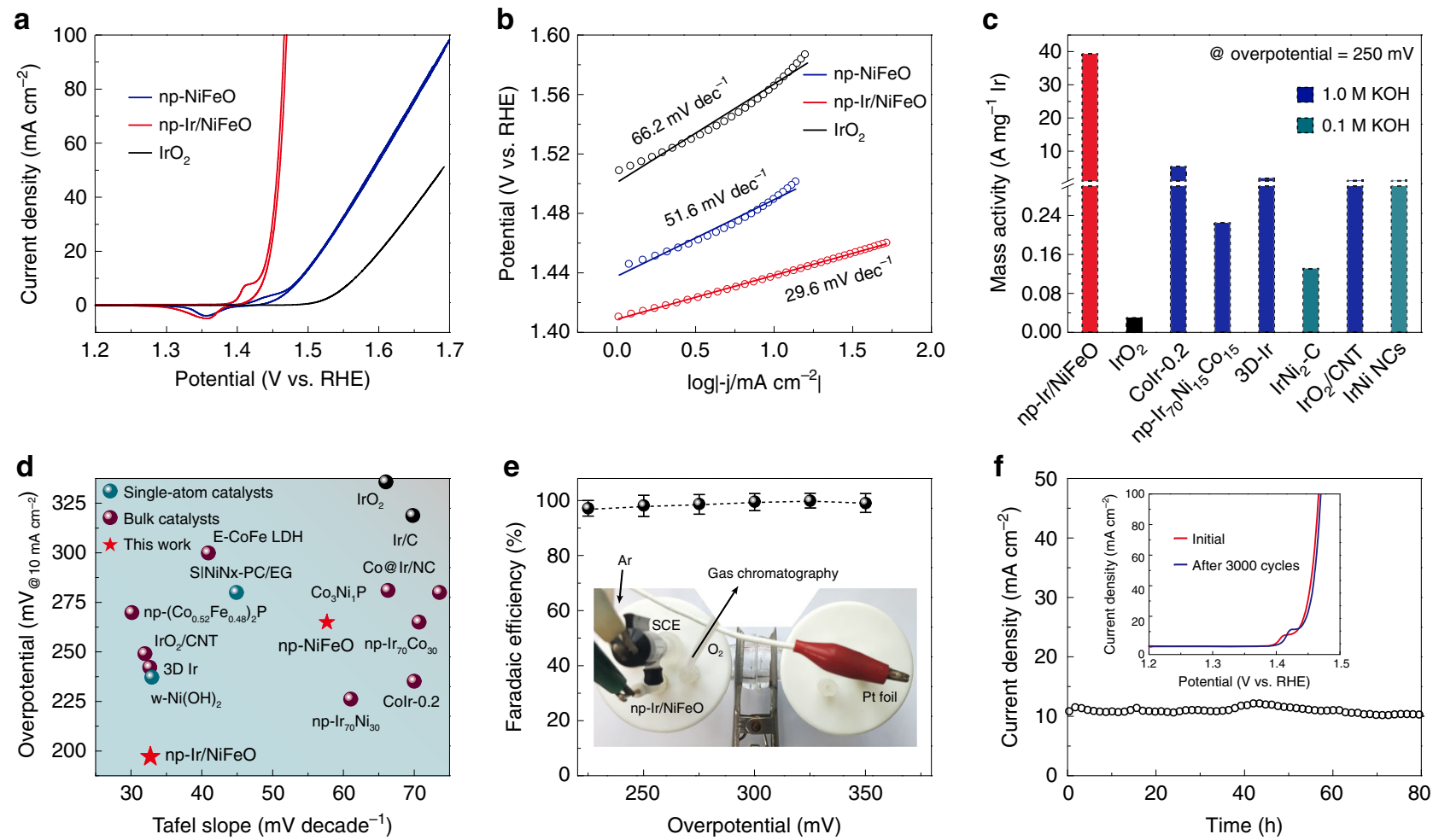

Fig. 2 Electrochemical OER performance. a OER polarization curves of np-NiFeO, np-Ir/NiFeO, and $1 \mathrm{rO}$. $\mathbf{b}$ Corresponding to Tafel plots of the presented data in (a). c The mass activities of $\mathrm{np}$ - $\mathrm{Ir} / \mathrm{NiFeO}, \mathrm{IrO}_{2}$, and state-of-the-art OER catalysts. $\mathbf{d}$ Comparison of Tafel slope and overpotential required to achieve $10 \mathrm{~mA} \mathrm{~cm}^{-2}$, with references all measured in alkaline medium. e Gas chromatography analyses for np-Ir/NiFeO. Inset shows the detail of Faradaic efficiency measurement. Error bars represent the standard deviation from multiple measurements. $\mathbf{f}$ The current density versus time (i-t) curves of $n p-I r /$ $\mathrm{NiFeO}$ recorded for $80 \mathrm{~h}$ at $1.43 \mathrm{mV}$ versus RHE. Inset shows the CV curves of $\mathrm{np}$-Ir/NiFeO before and after the acceleration durability test for 3000 cycles.

located at $2.81 \AA$ (denoted as peak I) could be viewed as a signal of $\mathrm{Ni}$ (oxy)hydroxides. Thus, the higher peak I in $\mathrm{np}-\mathrm{Ir} / \mathrm{NiFeO}$ further implies the formation of $\mathrm{Ni}$ (oxy)hydroxides ${ }^{7,10}$. In the case of Fe, XANES and FT-EXAFS results display similar phenomena to Ni (Supplementary Fig. 12c, d), which confirms that the surface Fe species have the same function and behavior as the surface $\mathrm{Ni}$ species.

Electrochemical oxygen evolution performance. The OER activities of $n p-\mathrm{NiFeO}$ and $\mathrm{np}-\mathrm{Ir} / \mathrm{NiFeO}$ were evaluated in a three-electrode set-up, along with commercial $\mathrm{IrO}_{2}$ as reference. Figure $2 \mathrm{a}$ shows the linear sweep voltammetry (LSV) of those catalysts in $\mathrm{O}_{2}$-saturated $\mathrm{KOH}$ solution. The np-Ir/NiFeO shows the highest performance among all samples, requiring overpotential of only 170 and $197 \mathrm{mV}$ to reach a current density of 1 (defined as onset overpotential) and $10 \mathrm{~mA} \mathrm{~cm}^{-2}$, significantly better than those of np-NiFeO and commercial $\mathrm{IrO}_{2}$ (Supplementary Fig. 13). Strikingly, np-Ir/ $\mathrm{NiFeO}$ electrode can achieve a high current density up to $300 \mathrm{~mA} \mathrm{~cm}^{-2}$ at $1.48 \mathrm{~V}$ versus reversible hydrogen electrode (RHE) because of enhanced transfer of mass (reactants and oxygen bubbles) (Supplementary Fig. 14), a large specific surface area, and efficient gas pumping of bicontinuous nanoporous structure (Supplementary Fig. 15). A small Tafel slope of $29.6 \mathrm{mV}$ per decade $\left(\mathrm{mV} \mathrm{dec}^{-1}\right)$ is measured for $\mathrm{np}-\mathrm{Ir} / \mathrm{NiFeO}$ (Fig. 2b), which is much smaller than $\mathrm{np}-\mathrm{NiFeO}$ $\left(51.6 \mathrm{mV} \mathrm{dec}^{-1}\right)$ and $\mathrm{IrO}_{2}\left(66.2 \mathrm{mV} \mathrm{dec}^{-1}\right)$. The lower Tafel slope and charge transfer resistance (Supplementary Fig. 16) of np-Ir/ $\mathrm{NiFeO}$ in relation to $\mathrm{np}-\mathrm{NiFeO}$ demonstrate that $\mathrm{np}-\mathrm{Ir} / \mathrm{NiFeO}$ is endowed with the favorable fast oxygen evolution kinetics by the introduction of isolated Ir atoms. Additionally, normalized to the Ir loading (0.1 wt\%, Supplementary Fig. 17), the mass activity of $\mathrm{np}-\mathrm{Ir} / \mathrm{NiFeO}$ for OER at an overpotential of $250 \mathrm{mV}$ is $39.3 \mathrm{~A} \mathrm{mg}^{-1}$, which is 131 times greater than that of the $\mathrm{IrO}_{2}$ catalyst $\left(0.30 \mathrm{~A} \mathrm{mg}^{-1}\right)$ (Supplementary Note 1 ). This result indicates that isolated Ir atoms anchored on $\mathrm{np}-\mathrm{NiFeO}$ support can maximize the catalytic activity, allowing significant cost reduction of the OER catalyst. Compared with previously reported OER catalysts, $\mathrm{np}-\mathrm{Ir} / \mathrm{NiFeO}$ shows the higher catalytic performance in terms of mass activity, overpotential at $10 \mathrm{~mA} \mathrm{~cm}^{-2}$, and Tafel slope in the alkaline solution (Fig. $2 \mathrm{c}, \mathrm{d}$ and Supplementary Tables 1, 2) $)^{5,17,22,27,37,43}$.

To elucidate the origin of the huge improvement of the OER activity, a CV method was utilized to measure the electrochemical double-layer capacitances $\left(\mathrm{C}_{\mathrm{dl}}\right)$ of corresponding catalysts for evaluating the electrochemically effective surface areas (ECSA $)^{18}$ Our results show a considerably larger $\mathrm{C}_{\mathrm{dl}}$ of $\mathrm{np}-\mathrm{Ir} / \mathrm{NiFeO}(21.4$ $\left.\mathrm{mF} \mathrm{cm}{ }^{-2}\right)$ compared with $\mathrm{np}-\mathrm{NiFeO}\left(14.9 \mathrm{mF} \mathrm{cm}^{-2}\right)$, manifesting that the new generated Ir sites act as the accessible active sites on $\mathrm{np}-\mathrm{Ir} / \mathrm{NiFeO}$ (Supplementary Fig. 18). The ECSA-normalized CV curves are employed to emphasize the intrinsic activity (Supplementary Note 2 and Supplementary Fig. 19). It is distinct that the ECSA-normalized current density of $n p-\mathrm{Ir} / \mathrm{NiFeO}$ is larger than that of np-NiFeO, indicating that the higher OER activity of np-Ir/NiFeO results from not only the increased ECSA but also the enhanced intrinsic activity. Besides, gas chromatography analyses show that np-Ir/NiFeO exhibits the $\mathrm{O}_{2}$ Faraday efficiency of near $100 \%$ under different applied potentials (Fig. 2e). During a chronoamperometry measurement at $1.43 \mathrm{~V}$ on np-Ir/ $\mathrm{NiFeO}$, the anodic current shows no evidence of degradation over $80 \mathrm{~h}$ (Fig. 2f). An accelerated CV cycling test was performed to verify this catalytic robustness with negligible shift of polarization curves after 3000 cycles (Fig. 2f inset). Further insights into the 

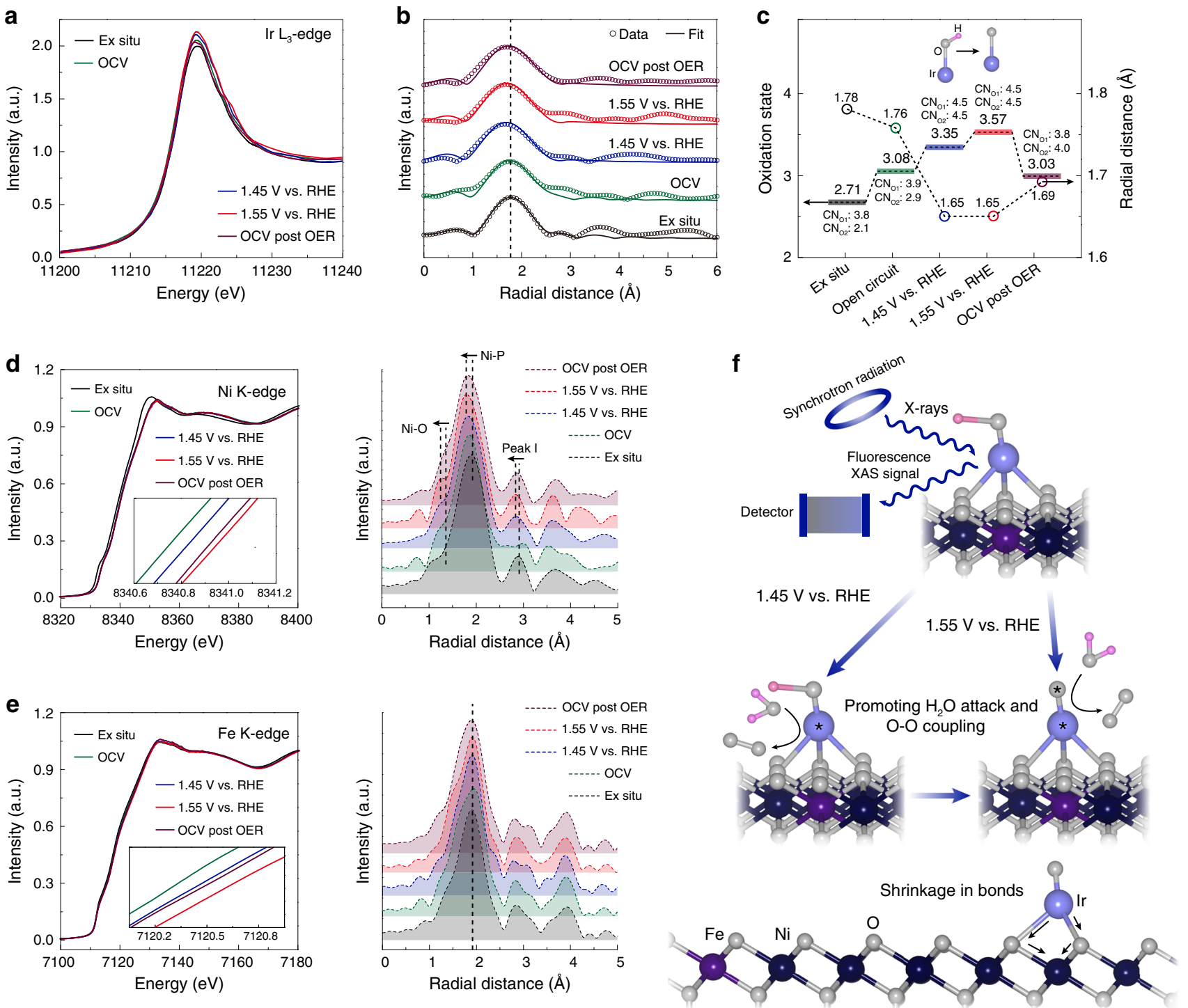

Fig. 3 Operando XAS characterizations. a Operando XANES spectra of np-Ir/NiFeO recorded at Ir $\mathrm{L}_{3}$-edge under different applied voltages from OCV to $1.55 \mathrm{~V}$ versus RHE in $1.0 \mathrm{M} \mathrm{KOH}$. b Corresponding first-shell (Ir-O) fitting of FT-EXAFS spectra for np-Ir/NiFeO. c The fitted oxidation states from the white line intensity analysis, the variation of Ir-O bond, and the FT-EXAFS curve-fitting analysis. d Operando XANES spectra of np-Ir/NiFeO recorded at Ni Kedge under different applied voltages from OCV to $1.55 \mathrm{~V}$ versus RHE in $1.0 \mathrm{M} \mathrm{KOH}$, as well as the corresponding FT-EXAFS spectra. e Operando XANES spectra of $\mathrm{np}-\mathrm{Ir} / \mathrm{NiFeO}$ recorded at Fe K-edge under different applied voltages from OCV to $1.55 \mathrm{~V}$ versus RHE in $1.0 \mathrm{M} \mathrm{KOH}$, as well as the corresponding FT-EXAFS spectra. $\mathbf{f}$ Schematic illustration of the OER mechanism determined by the operando XAS analysis of $\mathrm{np}-\mathrm{Ir} / \mathrm{NiFeO}$.

morphology for post-OER np-Ir/NiFeO by scanning electron microscopy (SEM) confirm the retaining of nanoporous structure (Supplementary Fig. 20). XAS measurement of np-Ir/NiFeO after long-time stability test displays some predictable changes (Supplementary Fig. 21), namely, all the metallic elements had relatively oxidized after long-time operation, but keeping the atomic dispersion of Ir atoms.

OER enhancement mechanism. To elucidate the origin of the outstanding OER catalytic performance and the structural advantages of $\mathrm{np}-\mathrm{Ir} / \mathrm{NiFeO}$, the dynamic changes in oxidation state and local coordination environment at OER-relevant potentials was probed by operando XAS analysis ${ }^{40}$. Figure $3 \mathrm{a}, \mathrm{b}$ display the normalized operando $\mathrm{Ir}_{3}$-edge XANES spectra and the corresponding FT-EXAFS spectra. Meanwhile, the fitted oxidation states from the white line intensity analysis, the variation of Ir-O bond, and the FT-EXAFS curve-fitting analysis are shown in Fig. 3c, Supplementary Figs. 22, 23, and Supplementary
Table 3. The analysis of Ir coordination shell was carried out by taking two backscattering paths, including $\mathrm{Ir}-\mathrm{O} 1$ (the $\mathrm{O}$ ligands and intermediate species) and $\mathrm{Ir}-\mathrm{O} 2$ (the chemisorption of $\mathrm{OH}^{-}$ and $\mathrm{H}_{2} \mathrm{O}$ ). Under the open-circuit voltage (denoted as OCV), np$\mathrm{Ir} / \mathrm{NiFeO}$ shows an increase of white line intensity in XANES spectrum compared with the ex-situ condition, resulting from the chemisorption of $\mathrm{OH}^{-}$and $\mathrm{H}_{2} \mathrm{O}^{30,44}$. This is also evidenced by the corresponding FT-EXAFS analysis, which shows the increase in the coordination number of $\mathrm{Ir}-\mathrm{O} 2$ (denoted as $\mathrm{CN}_{\mathrm{O} 2}$ ). During electrochemical OER (1.45 V versus RHE), a further increase of the white line intensity occurs in relation to the case under OCV, implying the formation of $\mathrm{Ir}$ intermediate species (such as $\mathrm{OOH}^{*}$ ) and the more chemisorption of $\mathrm{OH}^{-}$and $\mathrm{H}_{2} \mathrm{O}^{44,45}$. This variation of atomic coordination environment also reflects by the increase in the coordination number of $\mathrm{Ir}-\mathrm{O} 1$ (denoted as $\mathrm{CN}_{\mathrm{O} 1}$ ) and the $\mathrm{CN}_{\mathrm{O} 2}$ in the corresponding FT-EXAFS analysis. However, as the further increase of anodic potential $(1.55 \mathrm{~V}$ versus $\mathrm{RHE}), \mathrm{np}-\mathrm{Ir} / \mathrm{NiFeO}$ only displays the increase of white line 
intensity in XANES spectrum without the change of FT-EXAFS spectrum in comparison with that under $1.45 \mathrm{~V}$ versus RHE. This result manifests that the increase of the valance state of Ir under higher anodic potential was not result from the further chemisorption or the formation of intermediate species. Therefore, we propose a deprotonation process ( $\mathrm{Ir}-\mathrm{OH}$ to $\mathrm{Ir}-\mathrm{O}^{*}$ ) which occurs on Ir sites under higher applied potential. The deprotonation step will cause the oxidation of center atom while not change the atomic coordination environment ${ }^{4}$. The active oxygen species created during the deprotonation step play a critical role in efficient OER process, which serves as a synergistic site to promote the $\mathrm{H}_{2} \mathrm{O}$ attack and $\mathrm{O}-\mathrm{O}$ coupling ${ }^{6,46,47}$, resulting the faster OER kinetics under higher applied potential. When the potential restores back to OCV, the valance state of Ir for $\mathrm{np}-\mathrm{Ir} / \mathrm{NiFeO}$ can recover to its initial state, implying the restorability in the oxidation state of Ir. Additionally, it is clear that Ir exists as isolated atoms during the OER process without agglomeration, which is attributed to the shrinkage of $\mathrm{Ir}-\mathrm{O}$ bonds under realistic reaction conditions (Fig. 3b and Supplementary Table 3).

In the case of $\mathrm{Ni}$, the absorption-edge of $n p-\mathrm{Ir} / \mathrm{NiFeO}$ in XANES spectrum (Fig. 3d) displays an obvious positive shift under OCV condition in relation to the ex situ condition, manifesting the oxidation of the $\mathrm{Ni}$ species $^{48}$. Correspondingly, the main peak in the FT-EXAFS spectrum shows a low- $R$ shift, meaning the change of local atomic structure. During electrochemical OER (1.45 and $1.55 \mathrm{~V}$ versus RHE), the further oxidation behavior of $\mathrm{Ni}$ species is observed by the positive shift of the absorption-edge, indicating the transformation from hydroxides to oxyhydroxides in $\mathrm{np}-\mathrm{Ir} / \mathrm{NiFeO}$. Correspondingly, the FT-EXAFS spectrum shows the negative-shift of all peaks $(\mathrm{Ni}-\mathrm{O}, \mathrm{Ni}-\mathrm{P}$, and peak $\mathrm{I}$ ), especially for $\mathrm{Ni}-\mathrm{O}$ peak (splitting from main peak $)^{7}$, indicating the shrinkage of bonds in np-Ir/ $\mathrm{NiFeO}$. This shrinkage in $\mathrm{Ni}-\mathrm{O}$ bonds could further fix the isolated $\mathrm{Ir}$ atoms on the surface of $\mathrm{np}-\mathrm{Ir} / \mathrm{NiFeO}$, thus avoiding possible agglomeration during $\mathrm{OER}^{39}$. In particular, when the potential restores back to $\mathrm{OCV}$, the valance state of $\mathrm{Ni}$ cannot recover to its initial state, suggesting the irreversibility in the oxidation state of $\mathrm{Ni}$ during the OER process. In the case of $\mathrm{Fe}$, similar changes in XANES spectra (Fig. 3e) suggest the similar behavior of $\mathrm{Ni}$ and $\mathrm{Fe}$. Unlike $\mathrm{Ni}$, the main peak in Fe FT-EXAFS spectra exhibits constant radial distance. This could be resulted from the less content of $\mathrm{Fe}$ compared with $\mathrm{Ni}$ on the surface of $\mathrm{np}-\mathrm{Ir} / \mathrm{NiFeO}$, weakening the variation during OER process. Besides, further insights into the regulation effect of Ir atoms incorporation by controlled operando XAS measurement of np$\mathrm{Ir} / \mathrm{NiFeO}$ and $\mathrm{np}-\mathrm{NiFeO}$ confirm that more transformation from $\mathrm{Ni}(\mathrm{Fe})$ oxides or hydroxides to $\mathrm{Ni}(\mathrm{Fe})$ oxyhydroxides occurs on the surface of $n p-\mathrm{Ir} / \mathrm{NiFeO}$ than $\mathrm{np}-\mathrm{NiFeO}$, as evidenced by the greater shifts of the absorption-edge of $n p-\mathrm{Ir} / \mathrm{NiFeO}$ compared with that of $\mathrm{np}-\mathrm{NiFeO}$ under OER conditions (Supplementary Fig. 24) ${ }^{9}$. Considering the existence of strong metal-support interactions in $\mathrm{SACs}^{34,35}$, it is reasonable to believe that the charge transfer from the $\mathrm{Ni}(\mathrm{Fe})$ oxides or hydroxides to $\mathrm{Ir}$ atoms at the interfacial sites under realistic OER conditions is responsible for the facilitating of oxidation of $\mathrm{Ni}(\mathrm{Fe})$ oxides or hydroxides to $\mathrm{Ni}(\mathrm{Fe})$ oxyhydroxides, thus enabling an earlier onset of current for the OER 49,50 .

Based on the above operando XAS results, we propose a straightforward mechanism for the high OER activity and stability based on np-Ir/NiFeO (Fig. 3f). During electrochemical $\mathrm{OER}$, the $\mathrm{OH}$ ligands of $\mathrm{Ir}$ atoms undergo a deprotonation process and turn into new stable oxygen sites with the increase of applied anodic potential. Multiple active centers consisting of Ir sites and active oxygen species accelerate OER process under extremely conditions. Meanwhile, the shrinkage of the $\mathrm{Ni}(\mathrm{Fe})-\mathrm{O}$ bonds leads to a more stable surface structure, thus fixing the isolated Ir atoms and preventing agglomeration. In addition, it can be seen that the incorporation of Ir atoms promotes the formation of $\mathrm{Ni}(\mathrm{Fe})$ oxyhydroxides, further accelerating the reactions.

Theoretical investigations on OER activity. To further understand the OER mechanism of $n p-\mathrm{Ir} / \mathrm{NiFeO}$, theoretical investigations were performed based on DFT calculations ${ }^{27,51-57}$. The projected density of states (PDOS) results suggest that the introduction of Ir species endows new hybridized electronic states in $\mathrm{Ir} / \mathrm{NiFeO}$, resulting the widening of the total density of states (TDOS) near the Fermi level (Fig. 4a). This change is main attributed to the $\mathrm{Ni}$ and $\mathrm{Fe} 3 d$ orbitals $^{29}$, indicating that the $d$ electron domination of surface $\mathrm{Ni}$ and $\mathrm{Fe}$ atoms were optimize by the introduction of single-atom Ir, which leads to the increase of the effective charge concentration on the surface of $\mathrm{Ir} / \mathrm{NiFeO}$. Furthermore, the elementary reaction steps for the OER process in alkaline environments are demonstrated in Fig. 4b, Supplementary Figs. 25-29, Supplementary Note 3, and Supplementary Table 4. For pure NiFeO, Tafel slope shows that the $\mathrm{O}-\mathrm{O}$ coupling (the transition from $\mathrm{O}^{*}$ to $\mathrm{OOH}^{*}$ ) is the rate-determining step under alkaline conditions ${ }^{7}$. Thus, the large Gibbs free energy of the rate-determining step for $\mathrm{Ni}$ sites and $\mathrm{Fe}$ sites can slow down and even block the $\mathrm{O}_{2}$ evolution, resulting in an extremely sluggish OER kinetics (Fig. 4c). After the introduction of the Ir species, the Gibbs free energy of the rate-determining step for $\mathrm{Ni}$ sites and $\mathrm{Fe}$ sites dramatically decreased, suggesting a more favorable OER kinetics for $\mathrm{Ni}$ and Fe sites. Significantly, Ir sites in $\mathrm{Ir} / \mathrm{NiFeO}$ have the low Gibbs free energy of the rate-determining step than that of Ir sites on (001) face of Ir crystal, indicating that isolated $\mathrm{Ir}$ sites on $\mathrm{Ir}-\mathrm{NiFeO}$ are efficient active sites to catalyze OER (Supplementary Fig. 29). Afterward, we move onto the Ir-O moiety generating under realistic reaction conditions (Fig. $4 \mathrm{~d}$ ). It is found that the Gibbs free energy of the rate-determining step for the Ir-O moiety is substantially lower than that of other sites, which suggests the facilitation of nucleophilic $\mathrm{H}_{2} \mathrm{O}$ attack and $\mathrm{O}-\mathrm{O}$ coupling during OER process, in coincidence with the aforementioned operando XAS results. Consequently, the activation of $\mathrm{Ni}(\mathrm{Fe})$ oxyhydroxides and the formation of Ir-O moiety are responsible for the excellent OER activity of $\mathrm{Ir} / \mathrm{NiFeO}$.

\section{Discussion}

In summary, we developed a novel single-atom Ir incorporated into $\mathrm{Ni}(\mathrm{Fe})$ oxyhydroxides catalyst for OER, by utilizing the surface reconstruction of nanoporous $\mathrm{Ni}(\mathrm{Fe})$ phosphides. The asprepared $\mathrm{np}-\mathrm{Ir} / \mathrm{NiFeO}$ catalyst exhibited an outstanding OER performance in alkaline aqueous solutions, showing an OER overpotential of $197 \mathrm{mV}$ versus RHE to achieve a current density of $10 \mathrm{~mA} \mathrm{~cm}^{-2}$, an ultralow Tafel slope of $29.6 \mathrm{mV} \mathrm{dec}^{-1}$, a high mass activity of $39.3 \mathrm{~A} \mathrm{mg}^{-1}$ (131 times higher than that of the commercial $\mathrm{IrO}_{2}$ ), and excellent cycling stability. These impressive OER performances are comparable to, or even better than the values of state-of-the-art catalyst materials, including the commercial $\mathrm{IrO}_{2}$ catalyst. Through operando XAS analysis and DFT calculations, we find that the isolated Ir atoms vary from single active site to multiple actives through a deprotonation processes under realistic reaction conditions, leading to the excellent OER performance. The superior activity could be attributed to the Ir-O moiety, manifested in favorable formation of the $\mathrm{OOH}^{*}$ intermediate in the OER process. Meanwhile, the strong electron interaction between isolated Ir atoms and (oxy) hydroxides results in a more stable surface structure during OER process, confirming the excellent stability of isolated Ir atoms. Besides, the incorporation of Ir atoms could promote the formation of $\mathrm{Ni}(\mathrm{Fe})$ oxyhydroxides and arouse the intrinsic activity 
a

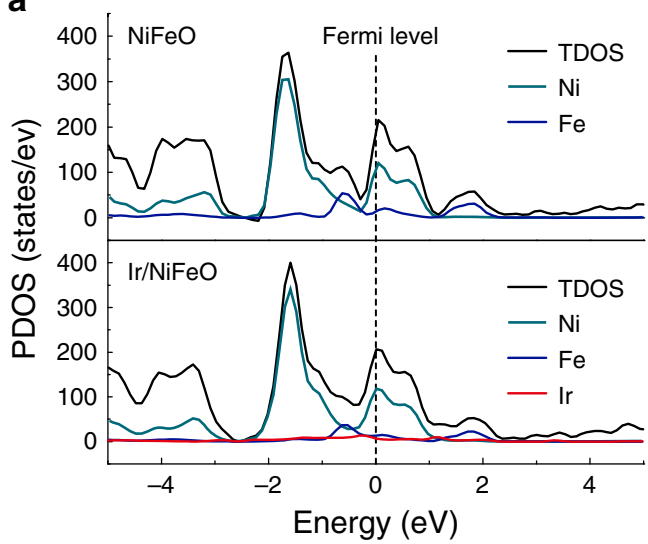

b

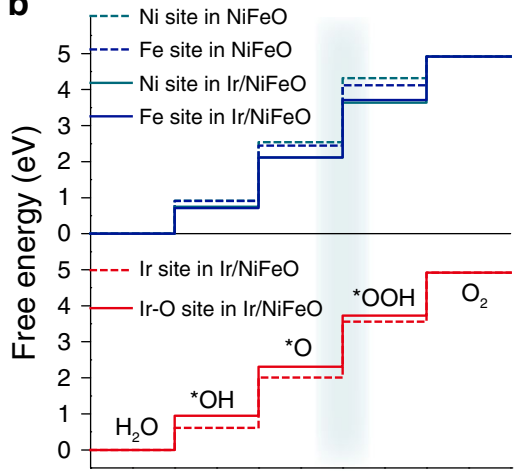

Reaction coordinate
C

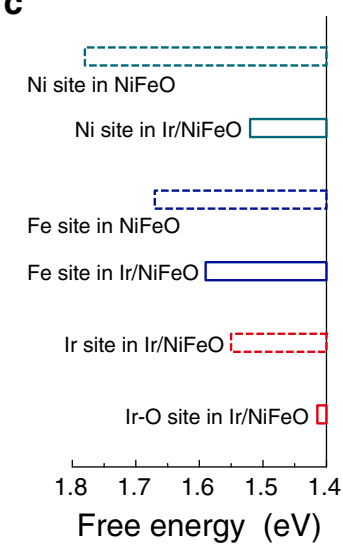

d

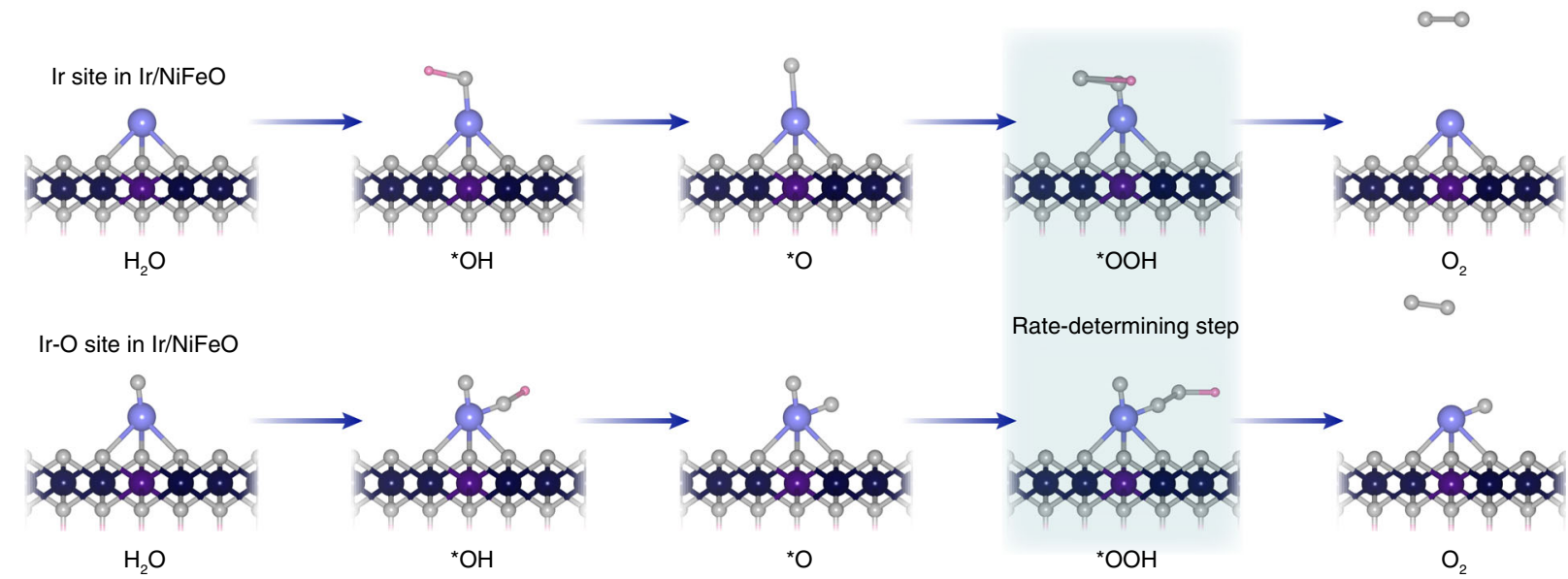

Fig. 4 DFT calculations. a Calculated PDOS of NiFeO and Ir/NiFeO. b Calculated free energy diagram of the OER. The blue box step is the rate-determining step. c The Gibbs free energy of the rate-determining step for different sites. d Schematic of the whole OER mechanism on Ir/NiFeO in the alkaline electrolyte.

of $\mathrm{Ni}(\mathrm{Fe})$ oxyhydroxides, further accelerating the reactions. Thus, this work presented herein highlight the importance of using operando XAS to identify the fine structure of the reconstructionderived components and deepen the understanding of the dynamic structure of single-atomic electrocatalysts in promoting OER activity.

\section{Methods}

Materials synthesis. The np-NiFeP was synthesized through electrochemically selective etching. Then, repeated CV scans was conducted to perform controllable deposition. Specifically, the $\mathrm{IrCl}_{3}$ powder $(1.0 \mathrm{mg})$ was added to $500 \mathrm{~mL}$ of $\mathrm{KOH}$ solution $(1.0 \mathrm{M})$ and stirred for $2 \mathrm{~h}$ to obtain the homogeneous mixture as an electrolyte. The deposition process was carried out by $750 \mathrm{CV}$ cycles with a voltage range from 0.075 to $-0.475 \mathrm{~V}$ versus RHE at a scan rate of $50 \mathrm{mV} \mathrm{s}^{-1}$. Finally, the self-reconstruction process was conducted in fresh $\mathrm{KOH}(1.0 \mathrm{M})$ by $50 \mathrm{CV}$ cycles with a voltage range from 1.0 to $1.6 \mathrm{~V}$ versus $\mathrm{RHE}$ at a scan rate of $50 \mathrm{mV} \mathrm{s}^{-1}$.

Characterization. XRD patterns were acquired by using a Bruker D8 advance $(\mathrm{Cu}$ Ka radiation, $\lambda=1.5418 \AA$ ). SEM measurements were performed on Zeiss Sigma HD (Oxford EDS). HRTEM images, HAADF-STEM images, and EDX mappings were obtained by using a JEM-ARM 200F. XPS date were achieved on Thermo Fisher Scientific ESCALAB250Xi spectrometer (Al Ka monochromatic). The specific surface area was collected based on the Brunauer-Emmet-Teller (BET) method using Micromeritics ASAP 2020. Inductively coupled plasma optical emission spectrometry (ICP-OES) was performed on Agilent 730.

Electrochemical testing. All electrochemical OER activity and durability were measured using an Ivium CompactStat.h electrochemical workstation with np-Ir/ $\mathrm{NiFeO}$ as the working electrode, a graphite sheet as the counter electrode, and a saturated calomel electrode (SCE) as the reference electrode in $\mathrm{O}_{2}$-saturated $1.0 \mathrm{M}$ $\mathrm{KOH}$. CV results were obtained in the range of 1.0 to $1.6 \mathrm{~V}$ versus RHE at a scan rate of $2 \mathrm{mV} \mathrm{s}^{-1}$. All potentials were converted to the RHE scale and iR-corrected by the resistance of the electrolyte. To minimize the masking effect of redox couple, we derived the Tafel plots and compared the OER activities using the cathodic sweep. The OER durability were measured by repeating the potential scan from 1.2 to $1.6 \mathrm{~V}$ versus RHE at a sweep rate of $50 \mathrm{mV} \mathrm{s}^{-1}$ with $3000 \mathrm{CV}$ cycles. Chronoamperometric characterization was performed at a potential of $1.43 \mathrm{~V}$ versus RHE for $80 \mathrm{~h}$.

Operando XAS measurements. The $\mathrm{Ir}_{3}$-edge, $\mathrm{Ni} \mathrm{K}$-edge, and Fe K-edge XAS spectra were measured at the beamline BL01C1 of National Synchrotron Radiation Research Center (NSRRC, Taiwan). Operando XAS measurements were performed using an Ivium CompactStat.h electrochemical workstation and a home-built cell equipped with a carbon rod counter electrode and a SCE reference electrode. The working electrode was prepared by dropping the catalyst ink on the carbon cloth. The fresh $\mathrm{KOH}$ electrolyte was bubbled with pure oxygen for $1 \mathrm{~h}$. For installation of operando XAS setup, the side of working electrode covered with Kapton film was faced to the incident X-rays. Simultaneously, the other side of working electrode was contacted with $\mathrm{KOH}$ electrolyte ${ }^{40}$. The XAS spectra were measured in the fluorescence mode at room temperature. During the operando experiments, the OER-relevant potentials of OCV, 1.45, and $1.55 \mathrm{~V}$ versus RHE were applied to this system. Acquired XAS data were processed with the ATHENA program.

DFT calculations. In this paper, the DFT $+\mathrm{U}$ calculations were performed with the Vienna ab initio simulation package (VASP) ${ }^{54}$. The exchange-correlation potential was treated by using the generalized gradient approximation (GGA) of Perdew-Burke-Ernzerhof (PBE) functional ${ }^{55,56}$. In the calculations, the value of $U$ is 3.8 for $\mathrm{Ni}, 4.3$ for $\mathrm{Fe}$, and 0 for $\mathrm{Ir}$ (no $U$ correction), which was selected according to the previous studies ${ }^{57}$. The (001)-terminated surface was selected in the $5 \times 5 \times 2$ $\gamma$-NiOOH and a vacuum slab of $15 \AA$ was added along the surface. The cutoff 
energy was set as $450 \mathrm{eV}$. During structural optimization and the self-consistent calculations, the Brillouin zone (BZ) was sampled by a Monkhorst-Pack k-mesh of $5 \times 5 \times 1$. The unit cell was optimized until the force and total energy were set to be $0.01 \mathrm{eV} / \AA$ and $10^{-5} \mathrm{eV}$, respectively.

\section{Data availability}

The data that support the findings of this study are available from the corresponding authors upon reasonable request.

Received: 15 January 2020; Accepted: 11 May 2020;

Published online: 01 June 2020

\section{References}

1. Chu, S. \& Majumdar, A. Opportunities and challenges for a sustainable energy future. Nature 488, 294-303 (2012).

2. Zhang, B. et al. Homogeneously dispersed multimetal oxygen-evolving catalysts. Science 352, 333-337 (2016).

3. Suen, N. T. et al. Electrocatalysis for the oxygen evolution reaction: recent development and future perspectives. Chem. Soc. Rev. 46, 337-365 (2017).

4. Wu, T. et al. Iron-facilitated dynamic active-site generation on spinel $\mathrm{CoAl}_{2} \mathrm{O}_{4}$ with self-termination of surface reconstruction for water oxidation. Nat. Catal. 2, 763-772 (2019).

5. Yan, J. et al. Single atom tungsten doped ultrathin alpha-Ni(OH $)_{2}$ for enhanced electrocatalytic water oxidation. Nat. Commun. 10, 2149 (2019).

6. Huang, Z.-F. et al. Chemical and structural origin of lattice oxygen oxidation in Co-Zn oxyhydroxide oxygen evolution electrocatalysts. Nat. Energy 4 , 329-338 (2019).

7. Song, F. et al. An unconventional iron nickel catalyst for the oxygen evolution reaction. ACS Cent. Sci. 5, 558-568 (2019).

8. Wang, H. Y. et al. In operando identification of geometrical-site-dependent water oxidation activity of spinel $\mathrm{Co}_{3} \mathrm{O}_{4}$. J. Am. Chem. Soc. 138, 36-39 (2016).

9. Kim, M. et al. Reducing the barrier energy of self-reconstruction for anchored cobalt nanoparticles as highly active oxygen evolution electrocatalyst. $A d v$. Mater. 31, 1901977 (2019).

10. Gorlin, M. et al. Oxygen evolution reaction dynamics, faradaic charge efficiency, and the active metal redox states of Ni-Fe oxide water splitting electrocatalysts. J. Am. Chem. Soc. 138, 5603-5614 (2016).

11. McCrory, C. C. et al. Benchmarking heterogeneous electrocatalysts for the oxygen evolution reaction. J. Am. Chem. Soc. 135, 16977-16987 (2013).

12. Diaz-Morales, O. et al. Guidelines for the rational design of Ni-based double hydroxide electrocatalysts for the oxygen evolution reaction. ACS Catal. 5, 5380-5387 (2015).

13. Suntivich, J. et al. A perovskite oxide optimized for oxygen evolution catalysis from molecular orbital principles. Science 334, 1383-1385 (2011).

14. Friebel, D. et al. Identification of highly active $\mathrm{Fe}$ sites in $(\mathrm{Ni}, \mathrm{Fe}) \mathrm{OOH}$ for electrocatalytic water splitting. J. Am. Chem. Soc. 137, 1305-1313 (2015).

15. Chen, P. et al. Strong-coupled cobalt borate nanosheets/graphene hybrid as electrocatalyst for water oxidation under both alkaline and neutral conditions. Angew. Chem. Int. Ed. 55, 2488-2492 (2016).

16. Masa, J. et al. Amorphous cobalt boride $\left(\mathrm{Co}_{2} \mathrm{~B}\right)$ as a highly efficient nonprecious catalyst for electrochemical water splitting: oxygen and hydrogen evolution. Adv. Energy Mater. 6, 1502313 (2016).

17. Tan, Y. et al. Versatile nanoporous bimetallic phosphides towards electrochemical water splitting. Energy Environ. Sci. 9, 2257-2261 (2016).

18. Yu, F. et al. High-performance bifunctional porous non-noble metal phosphide catalyst for overall water splitting. Nat. Commun. 9, 2551 (2018).

19. Huang, C. et al. Unveiling the active sites of Ni-Fe phosphide/metaphosphate for efficient oxygen evolution under alkaline conditions. Chem. Commun. 55, 7687-7690 (2019).

20. Laursen, A. B. et al. Climbing the volcano of electrocatalytic activity while avoiding catalyst corrosion: $\mathrm{Ni}_{3} \mathrm{P}$, a hydrogen evolution electrocatalyst stable in both acid and alkali. ACS Catal. 8, 4408-4419 (2018).

21. Hu, F. et al. Amorphous metallic NiFeP: a conductive bulk material achieving high activity for oxygen evolution reaction in both alkaline and acidic media. Adv. Mater. 29, 1606570 (2017).

22. Wang, T. et al. NiFe (Oxy) hydroxides derived from NiFe disulfides as an efficient oxygen evolution catalyst for rechargeable $\mathrm{Zn}$-Air batteries: The Effect of Surface S Residues. Adv. Mater. 30, 1800757 (2018).

23. Zhou, W. et al. $\mathrm{Ni}_{3} \mathrm{~S}_{2}$ nanorods/Ni foam composite electrode with low overpotential for electrocatalytic oxygen evolution. Energy Environ. Sci. 6, 2921-2924 (2013).
24. Zhu, Y. et al. Operando unraveling the structural/chemical stability of Psubstituted $\mathrm{CoSe}_{2}$ electrocatalysts toward hydrogen/oxygen evolution reactions in alkaline electrolyte. ACS Energy Lett. 4, 987-994 (2019).

25. Xia, C. et al. Selenide-based electrocatalysts and scaffolds for water oxidation applications. Adv. Mater. 28, 77-85 (2016).

26. Qiao, B. et al. Single-atom catalysis of $\mathrm{CO}$ oxidation using $\mathrm{Pt}_{1} / \mathrm{FeO}_{\mathrm{x}}$. Nat. Chem. 3, 634-641 (2011).

27. Hou, Y. et al. Atomically dispersed nickel-nitrogen-sulfur species anchored on porous carbon nanosheets for efficient water oxidation. Nat. Commun. 10 1392 (2019)

28. Cao, L. et al. Dynamic oxygen adsorption on single-atomic Ruthenium catalyst with high performance for acidic oxygen evolution reaction. Nat. Commun. 10, 4849 (2019).

29. Zhang, H. et al. Unveiling the activity origin of electrocatalytic oxygen evolution over isolated $\mathrm{Ni}$ atoms supported on a $\mathrm{N}$-doped carbon matrix. $A d v$ Mater. 31, 1904548 (2019).

30. Bai, L. et al. A cobalt-iron double-atom catalyst for the oxygen evolution reaction. J. Am. Chem. Soc. 141, 14190-14199 (2019).

31. Yao, Y. et al. Engineering the electronic structure of single atom Ru sites via compressive strain boosts acidic water oxidation electrocatalysis. Nat. Catal. 2, 304-313 (2019).

32. Zhao, Y. et al. Stable iridium dinuclear heterogeneous catalysts supported on metal-oxide substrate for solar water oxidation. Proc. Natl Acad. Sci. USA 115, 2902-2907 (2018)

33. Jiang, H. et al. Structural self-reconstruction of catalysts in electrocatalysis. Acc. Chem. Res. 51, 2968-2977 (2018).

34. Wang, A., Li, J. \& Zhang, T. Heterogeneous single-atom catalysis. Nat. Rev. Chem. 2, 65-81 (2018).

35. Chen, Y. et al. Single-atom catalysts: synthetic strategies and electrochemical applications. Joule 2, 1242-1264 (2018)

36. Tan, Y. et al. 3D Nanoporous metal phosphides toward high-efficiency electrochemical hydrogen production. Adv. Mater. 28, 2951-2955 (2016).

37. Zhang, Y. et al. Atomic iridium incorporated in cobalt hydroxide for efficient oxygen evolution catalysis in neutral electrolyte. Adv. Mater. 30, 1707522 (2018).

38. Ghiasi, M. et al. Charge-transfer effect in hard $x$-ray $1 s$ and $2 p$ photoemission spectra: LDA+DMFT and cluster-model analysis. Phys. Rev. B 100, 075146 (2019).

39. Li, P. et al. Boosting oxygen evolution of single-atomic ruthenium through electronic coupling with cobalt-iron layered double hydroxides. Nat. Commun. 10, 1711 (2019).

40. Jiang, K. et al. Single platinum atoms embedded in nanoporous cobalt selenide as electrocatalyst for accelerating hydrogen evolution reaction. Nat. Commun. 10, 1743 (2019).

41. Chen, Y. et al. Optimizing reaction paths for methanol synthesis from $\mathrm{CO}_{2}$ hydrogenation via metal-ligand cooperativity. Nat. Commun. 10, 1885 (2019).

42. Nam, G. et al. A ternary $\mathrm{Ni}_{46} \mathrm{Co}_{40} \mathrm{Fe}_{14}$ nanoalloy-based oxygen electrocatalyst for highly efficient rechargeable zinc-air batteries. Adv. Mater. 30, 1803372 (2018).

43. Zhao, Y. et al. 3D nanoporous iridium-based alloy microwires for efficient oxygen evolution in acidic media. Nano Energy 59, 146-153 (2019).

44. Wu, G. et al. A general synthesis approach for amorphous noble metal nanosheets. Nat. Commun. 10, 4855 (2019).

45. Tian, X. et al. Engineering bunched Pt-Ni alloy nanocages for efficient oxygen reduction in practical fuel cells. Science 366, 850-856 (2019).

46. Hintermair, U. et al. Precursor transformation during molecular oxidation catalysis with organometallic iridium complexes. J. Am. Chem. Soc. 135, 10837-10851 (2013).

47. Diaz-Morales, O. et al. Electrochemical and spectroelectrochemical characterization of an iridium-based molecular catalyst for water splitting: turnover frequencies, stability, and electrolyte effects. J. Am. Chem. Soc. 136, 10432-10439 (2014)

48. Yang, H. B. et al. Atomically dispersed $\mathrm{Ni}(\mathrm{i})$ as the active site for electrochemical $\mathrm{CO}_{2}$ reduction. Nat. Energy 3, 140-147 (2018).

49. Seitz, L. C. et al. Enhancement effect of noble metals on manganese oxide for the oxygen evolution reaction. J. Phys. Chem. Lett. 6, 4178-4183 (2015).

50. Zhang, $\mathrm{H}$. et al. Enhanced interactions between gold and $\mathrm{MnO}_{2}$ nanowires for water oxidation: a comparison of different chemical and physical preparation methods. ACS Sustain. Chem. Eng. 3, 2049-2057 (2015).

51. Koper, M. T. M. Thermodynamic theory of multi-electron transfer reactions: Implications for electrocatalysis. J. Electroanal. Chem. 660, 254-260 (2011).

52. Man, I. C. et al. Universality in oxygen evolution electrocatalysis on oxide surfaces. ChemCatChem 3, 1159-1165 (2011).

53. Rossmeisl, J. et al. Electrolysis of water on oxide surfaces. J. Electroanal. Chem. 607, 83-89 (2007). 
54. Kresse, G. \& Furthmüller, J. Efficient iterative schemes for ab initio totalenergy calculations using a plane-wave basis set. Phys. Rev. B 54, 11169-11186 (1996).

55. Perdew, J. P., Burke, K. \& Ernzerhof, M. Generalized gradient approximation made simple. Phys. Rev. Lett. 77, 3865-3868 (1996).

56. Kresse, G. \& Joubert, D. From ultrasoft pseudopotentials to the projector augmented-wave method. Phys. Rev. B 59, 1758-1775 (1999)

57. $\mathrm{Bi}, \mathrm{Y}$. et al. Understanding the incorporating effect of $\mathrm{Co}^{2+/} \mathrm{Co}^{3+}$ in $\mathrm{NiFe}$ layered double hydroxide for electrocatalytic oxygen evolution reaction. J. Catal. 358, 100-107 (2018).

\section{Acknowledgements}

The authors gratefully acknowledge financial support by the National Natural Science Foundation of China (Grant No. 51771072), the Youth 1000 Talent Program of China, the Fundamental Research Funds for the Central Universities, and Hunan University State Key Laboratory of Advanced Design and Manufacturing for Vehicle Body Independent Research Project (No. 71860007).

\section{Author contributions}

Y.W.T. conceived and supervised this study. K.J. and Y.Q.Y. carried out materials fabrication, $\mathrm{XRD} / \mathrm{SEM} / \mathrm{XPS} / \mathrm{ICP} / \mathrm{BET}$ characterizations, and electrochemical measurements. P.L. conducted the TEM characterizations. M.L. performed the DFT calculations and computational models. K.J., M.P., Y.R. L., T.S.C., and F.M.F.de G. contributed to the XAS measurements and analyses of the XAS experiments results. Y.W.T. and K.J. wrote the paper. All authors contributed to discussions and paper review.

\section{Competing interests}

The authors declare no competing interests.

\section{Additional information}

Supplementary information is available for this paper at https://doi.org/10.1038/s41467020-16558-1.

Correspondence and requests for materials should be addressed to Y.T.

Peer review information Nature Communications thanks Mårten Ahlquist, and the other, anonymous, reviewer(s) for their contribution to the peer review of this work. Peer reviewer reports are available

Reprints and permission information is available at http://www.nature.com/reprints

Publisher's note Springer Nature remains neutral with regard to jurisdictional claims in published maps and institutional affiliations.

\section{(c) (i)}

Open Access This article is licensed under a Creative Commons Attribution 4.0 International License, which permits use, sharing, adaptation, distribution and reproduction in any medium or format, as long as you give appropriate credit to the original author(s) and the source, provide a link to the Creative Commons license, and indicate if changes were made. The images or other third party material in this article are included in the article's Creative Commons license, unless indicated otherwise in a credit line to the material. If material is not included in the article's Creative Commons license and your intended use is not permitted by statutory regulation or exceeds the permitted use, you will need to obtain permission directly from the copyright holder. To view a copy of this license, visit http://creativecommons.org/ licenses/by/4.0/.

(C) The Author(s) 2020 